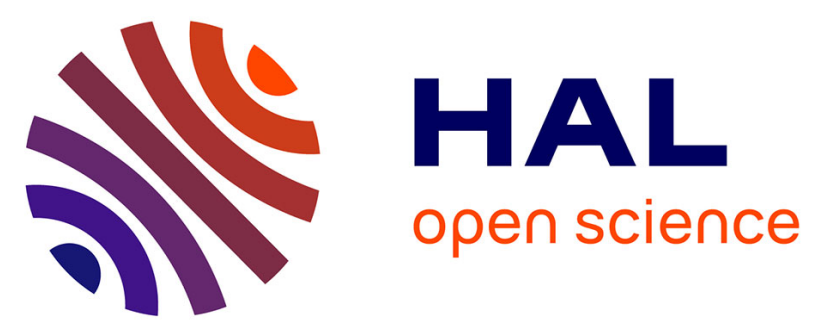

\title{
EXCITON-DEFECT INTERACTION IN THE ALKALI HALIDES
}

A. Nouailhat, E. Mercier, G. Guillot

\section{To cite this version:}

A. Nouailhat, E. Mercier, G. Guillot. EXCITON-DEFECT INTERACTION IN THE

ALKALI HALIDES. Journal de Physique Colloques, 1976, 37 (C7), pp.C7-492-C7-494. 10.1051/jphyscol:19767111 . jpa-00216833

\section{HAL Id: jpa-00216833 https://hal.science/jpa-00216833}

Submitted on 1 Jan 1976

HAL is a multi-disciplinary open access archive for the deposit and dissemination of scientific research documents, whether they are published or not. The documents may come from teaching and research institutions in France or abroad, or from public or private research centers.
L'archive ouverte pluridisciplinaire HAL, est destinée au dépôt et à la diffusion de documents scientifiques de niveau recherche, publiés ou non, émanant des établissements d'enseignement et de recherche français ou étrangers, des laboratoires publics ou privés. 


\title{
EXCITON-DEFECT INTERACTION IN THE ALKALI HALIDES
}

\author{
A. NOUAILHAT, E. MERCIER and G. GUILLOT \\ Laboratoire de Physique de la Matière $\left({ }^{*}\right)$ \\ Institut National des Sciences Appliquées de Lyon \\ 20, avenue Albert-Einstein, 69621 Villeurbanne Cedex, France
}

\begin{abstract}
Résumé. - On mesure l'interaction exciton-défaut dans $\mathrm{KI}$ entre $4 \mathrm{~K}$ et $77 \mathrm{~K}$ par l'étude du comportement de la luminescence intrinsèque dans les cristaux irradiés en utilisant conjointement comme excitation les UV et un faisceau électronique de moyenne énergie (60 keV). Ce dernier est aussi utilisé pour créer les défauts. On en déduit que la distance d'interaction augmente en dessous de $40 \mathrm{~K}$ uniquement pour les excitons créés par UV dans le premier pic d'absorption. Ce résultat est interprété par une diffusion athermique de l'exciton non relaxé à très basse température.
\end{abstract}

Abstract. - Exciton-defect interaction in $\mathrm{KI}$ between $4 \mathrm{~K}$ and $77 \mathrm{~K}$ is measured through the study of the intrinsic luminescence of irradiated crystals excited by both UV and medium energy $(60 \mathrm{keV})$ electron irradiation. The latter is also used to create the defects. We find that the interaction range increases below $40 \mathrm{~K}$ only for excitons created by UV absorption in the first exciton peak. This result is interpreted by the athermal diffusion of the non relaxed exciton at very low temperature.

The problem of energy transfer by exciton diffusion at low temperature in the alkali halides is always an open one. Much work has been done in this field, mainly on crystals doped with luminescent ions of $\mathrm{ns}^{2}$ type. Varying the experiments' parameters such as excitation wavelength in the first excitonic peak, activator's concentration and temperature, people were able to conclude either to the ability for the exciton to diffuse or to the absence of exciton diffusion in these crystals. The main reason for this ambiguity is the fact that ions like $\mathrm{Tl}^{+}, \mathrm{Ga}^{+}, \mathrm{In}^{+}$, give rise, in the crystal, to an absorption band ( $D$ band) attributed to the exciton localized close to the ion, and this band lies in the immediate vicinity of the first exciton peak of the host crystal. The interpretation of some results in these experiments is quite complicated because of the partial overlapping of the long wavelength side of the crystal excitonic band and the short wavelength side of the $\mathrm{D}$ band, and a doubt subsists as to whether the luminescence of the ion is a directly excited one or a host sensitized one.

Recent work by Nishimura et $a l$. on $\mathrm{KI}$, both pure [1] and doped with $\mathrm{ns}^{2}$ ions [2] gives account for the luminescent properties of $\mathrm{KI}:$ in this case of pure $\mathrm{KI}$, the relaxation to the relaxed states of the exciton is described as a thermally activated process. This implies the existence of a free exciton state before the relaxation. In this state, the exciton could diffuse to the luminescent impurities in the case of the doped crystals. At low temperature, the relaxation occurs by a tunneling process. Before that, Kamejima et al. [3],

(*) Equipe de recherche associée au C. N. R. S. investigating systematically $\mathrm{ns}^{2}$ ions luminescence in $\mathrm{KI}$, had concluded that no energy transfer took place between the exciton and luminescent ions : their luminescence had been attributed to the overlapping of the $\mathrm{D}$ band with exciton absorption region. The energy transfer to $\mathrm{Tl}^{+}$ions in $\mathrm{KI}: \mathrm{Tl}$ is partly attributed to exciton diffusion by Donahue [4] especially in the case of excitation in the first exciton peak. This result is obtained considering that transfer by resonance is not sufficient to explain the sensitization of large interaction volumes. Experiments were also performed using no luminescent ions : this is the case here and in the paper by Hayashi et al. [5] where point defects are used as quenching centers for the intrinsic luminescences.

We have shown on a previous paper [6] that the interstitials are the centers responsible for the quenching of luminescence in the alkali halides and we have established the dependence of the luminescence yield versus defect concentration : $I / I_{0}=\exp -\beta n(\mathrm{~V})$, where :

$I_{0} \quad$ : intensity of luminescence of the virgin crystal $I$ : intensity of luminescence of the irradiated crystal in the same conditions of excitation $n(\mathrm{~V})$ : concentration of defects

$\beta \quad$ : phenomenological parameter function of the excitation type, observed luminescence and temperature of defect creation.

$\beta$ defines an interaction volume where the defects cause the exciton to deexcite by a non radiative mechanism. This paper's purpose is to show that exciton diffusion can account for the quenching of luminescence depending on excitation type and temperature. 
Experimental apparatus (Fig. 1). - We have used an electron irradiation facility allowing coloration of the crystal by medium energy electrons $(60 \mathrm{keV})$ described in [7]. The exact concentration of defects can be measured up to a few $10^{19} \mathrm{~cm}^{-3}$. The electron beam is also used to make cathodoluminescence measurements on the irradiated crystal; optical excitation in the high energy side of the first exciton peak $(205 \mathrm{~nm})$ can be performed simultaneously at any defect concentration. The intensity of the intrinsic luminescence $\pi(380 \mathrm{~nm}$ for electronic excitation and $380 \mathrm{~nm}+410 \mathrm{~nm}$ for UV excitation [1]) is measured at $90^{\circ}$. The temperature range is from $4 \mathrm{~K}$ to $77 \mathrm{~K}$. The defects, $\mathrm{F}$ and $\mathrm{V}_{4}$ centers [8] $\left({ }^{1}\right)$, are always created at $77 \mathrm{~K}$ by the electron beam. Below this temperature, cathodoluminescence measurements can take place without modification of the number of defects, because the coloring power of the radiation becomes very small and we use a low density beam for cathodoluminescence excitation.

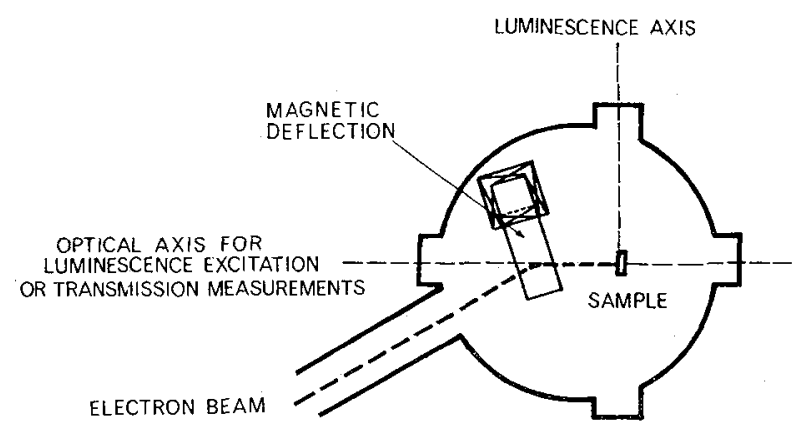

FIG. 1. - Experimental arrangement.

So, our apparatus allows us to make comparisons between photo- and cathodoluminescence yields in identical and well known conditions of defects iconcentrations and temperature. Optical excitation is used to form bound excitons whereas electron irradiation, as ionizing radiation, creates decorrelated electron-hole pairs.

Experimental results. - i) At the temperature of $77 \mathrm{~K}$, we have measured the quenching of both cathodo- and photoluminescence which is the ratio of the luminescence of the virgin crystal, $I_{0}$, to that of the crystal containing $n(\mathrm{~F}) \mathrm{F}$ centers per $\mathrm{cm}^{3}$. We have found that the quenching law is the same in both cases up to a few $10^{18} \mathrm{~F}$ centers per $\mathrm{cm}^{3}$ (Fig. 2).

ii) We have measured the quenching of both cathodo- and photoluminescence in the temperature range of $4 \mathrm{~K}$ to $77 \mathrm{~K}$ for a determined concentration of point defects created at $77 \mathrm{~K}$.

We find that for the cathodoluminescence, the quenching $I_{0} / I$ is practically constant between $4 \mathrm{~K}$ and $77 \mathrm{~K}$. For the photoluminescence, it varies significantly in the same temperature range, especially

(1) The number of $F$ centers is proportional to the number of $\mathrm{V}_{4}$ centers. For experimental convenience, we determine the concentration of defects by measurement of the $F$ band.

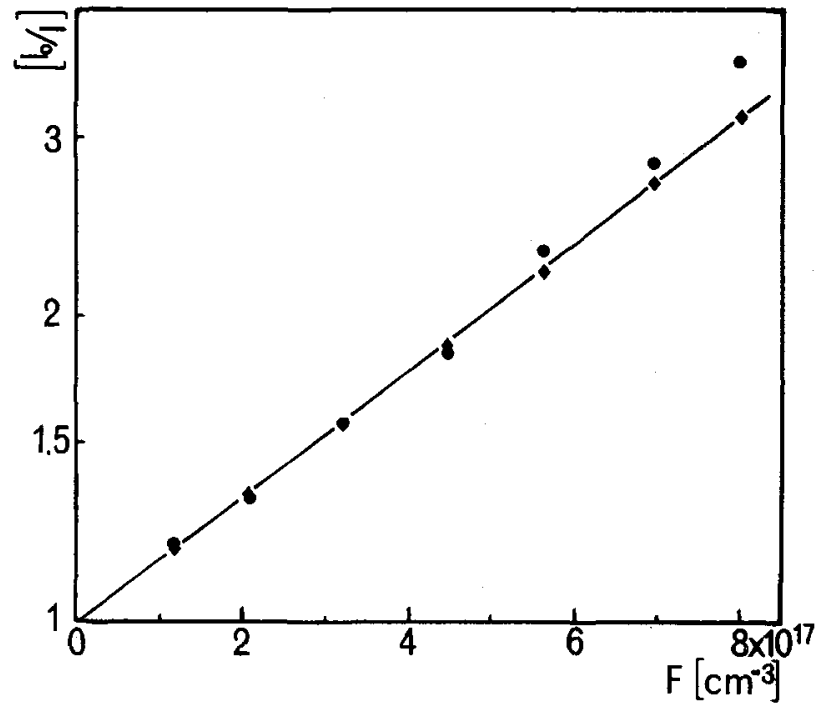

FIG. 2. - Comparison of the quenching of the $\pi$ luminescence in $\mathrm{KI}$ under electron ( $)$ and $\mathrm{UV}(\boldsymbol{)})$ excitation at $77 \mathrm{~K}$ as a function of $F$ center concentration (electron irradiation $55 \mathrm{kV}$, $3 \mu \mathrm{A} / \mathrm{cm}^{2}$; UV excitation at $205 \mathrm{~nm}$ ).

between $30 \mathrm{~K}$ and $50 \mathrm{~K}$, being about 2.5 times larger than for the electron excitation at $4 \mathrm{~K}$ and the same at $77 \mathrm{~K}$ (Fig. 3).

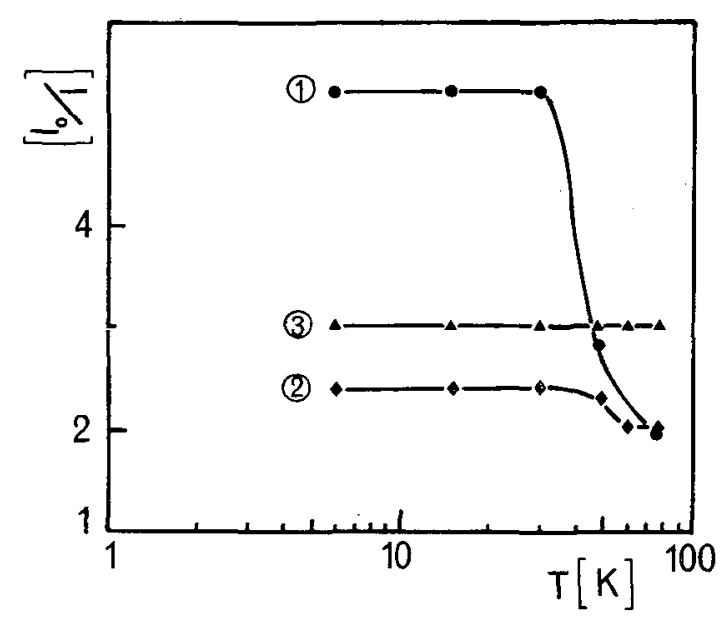

FIG. 3. - Quenching as a function of temperature of : - the $\pi$ luminescence in pure KI : (1) under UV excitation at $205 \mathrm{~nm}$ (the sum of both $\pi$ components at $380 \mathrm{~nm}$ and $410 \mathrm{~nm}$ is considered); (2) under electron irradiation ; - the luminescence of the localized exciton on $\mathrm{Tl}^{+}$in $\mathrm{KI}: \mathrm{Tl}(1 \% \mathrm{Tl})$ by $\mathrm{UV}$ excitation in the $\mathrm{D}$ band : (3). (The defects created by electron irradiation at $77 \mathrm{~K}$ are not in the same concentration in $[(1),(2)]$ and (3)).

iii) We have measured the quenching of the $425 \mathrm{~nm}$ luminescence of $\mathrm{KI}: \mathrm{Tl}(1 \%)$ colored at $77 \mathrm{~K}$ at a determined concentration of defects, as a function of the temperature between $4 \mathrm{~K}$ and $77 \mathrm{~K}$. The excitation was in the $\mathrm{D}$ band, which is the exciton localized close to the thallium. We have found that this quenching does not vary repetition with temperature (Fig. 3).

The results on photoluminescence are close to those by Hayashi et al. [5] who performed quenching measurement in the same range of temperature on pure $\mathrm{KI}$ colored by UV from an unfiltered deuterium lamp. 
We notice that their results [5] give a much larger value for the exciton-defect interaction than ours. This difference is certainly due to an error in the defect concentration estimation obtained with UV irradiation; it is indeed difficult to know the profile of coloration obtained with an unfiltered deuterium lamp, whereas with electron irradiation, the defect concentration is homogeneous and precisely known [9].

Discussion. - The decrease of the quenching of luminescence with temperature can be interpreted if the exciton's relaxation process into the luminescent states is a thermally activated one, and if the exciton diffuses in its non relaxed state. So, the higher the temperature, the smaller the diffusion length, i. e. the lower the probability of collision between the exciton and a quenching center. These considerations are only valid for free excitons, i. e. created by photon absorption in the first exciton peak. In the case of electronhole pairs, created by any ionizing radiation, the hole becomes immediately self trapped $\left({ }^{2}\right)$ until the capture of an electron, giving rise to an exciton unable to diffuse. Only the exciton-defect pairs formed statistically close enough, can interact to give non radia-

(2) The concept of hole migration in an unrelaxed state seems to be considered by russian authors [10] but we have no evidence in our experiments of this fact. tive recombination of the exciton independent of temperature. At $4 \mathrm{~K}$, a free exciton can diffuse over quite large distances in its metastable state, having a large probability of being quenched by encounter with an interstitial, so the quenching is important. At intermediate temperatures, we see that the diffusion length of the exciton decreases between $30 \mathrm{~K}$ and $50 \mathrm{~K}$, giving rise to the observed decreasing quenching of luminescence. At $77 \mathrm{~K}$, where the self trapping probability of the exciton is large, a free exciton cannot move before self trapping and the quenching is the same for both type of excitons, created either as free exciton or as ionized exciton.

In the case of the luminescence of thallium excited in the D band, where the exciton is formed in the immediate vicinity of the thallium ion, we observe no temperature dependence of the luminescence yield; diffusion is not necessary for energy transfer to the thallium. The observed quenching, which increases with defect concentration, may be accounted for by a statistical formation of close interstitial-thallium pairs which do not luminesce.

Our results in KI lead to suggest that exciton diffusion is an athermal process possible at low temperature before relaxation. That is the same conclusion as those of some papers about other alkali halides, mainly NaI [11] [12] RbI [13] CsBr [14] KBr [15].

\section{References}

[1] Nishimura, H., J. Phys. Soc. Japan 38 (1975) 450.

[2] Nishrmura, H. and Tomura, M., J. Phys. Soc. Japan 39 (1975) 390.

[3] Kamejima, T., Shionoya, S. and Fukuda, A., J. Phys. Soc. Japan 30 (1971) 1124.

[4] Donahue, J. M. Jr, Ph. D. Thesis, University of Rochester (1970).

[5] Hayashi, T., Ohata, T. and Koshino, S., Solid State Commun. 17 (1975) 945 ; Solid State Commun. 18 (1976) 807.

[6] Gulllot, G., Nouailhat, A., Mercier, E. and Pinard P., J. Lumin. 12/13 (1976) 327.

[7] Mercier, E., Guillot, G. and Nouailhat, A., in Revue Phys. Appl, to be published.
[8] Goldstern, F. T., Phys. Status Solidi 20 (1967) 379.

[9] Herrmann, F. and Pinard, P., J. Phys. C 3 (1970) 1037.

[10] Aluker, E. D., Rulev, Y. P., Stankevich, V. A. and Chernov, S. A., Izv. Akad. Nauk SSSR, Ser. Fiz. 38 (1974) 1230.

[11] Fontana, M. P., Blume, H. and Van Sciver, W. I., Phys. Status Solidi 29 (1968) 159.

[12] Blume, H., Fontana, M. P. and Van Sciver, W. I., Phys. Status Solidi 31 (1969) 133.

[13] Hattori, A., Tomura, M. and Nishimura, H., J. Phys. Soc. Japan 31 (1971) 611.

[14] Vasil'chenko, E. A., Lushchik, N. E. and Lushchik, Ch. J. Lumin. 5 (1972) 117.

[15] Vasil'chenko, E. A., Lushchik, N. E. and Soovik, Kh. A., Izv. Akad. Nauk SSSR, Ser. Fiz. 38 (1974) 1267.

\section{DISCUSSION}

M. C. WintersGill. - Had the speaker considered the possibility that under UV irradiation at low temperature might give rise to sputtering thus providing a radiationless method of exciton decay ?

A. Nouallhat. - No, because of the reversibility of the phenomena. For instance, the variation of the quenching versus temperature is a reversible phenomenon.

J. ARENDS. - How is the behavior of luminescence in $\mathrm{KI}$ if we compare X-irradiation and electron irradiation related to the defect concentration?

A. Nouailhat. - The behavior is the same for both irradiations.
J. D. Comrns. - If the presence of interstitial defects provides a mechanism whereby the energy of the radiation produced exciton may be dissipated, then one would expect this to influence the rate of F-center production as a function of irradiation time, i. e. the shape of the growth curve. Have you made measurements of this type or would expect such an effect to occur?

A. Nouailhat. - No, the concentration of defects has no effect on the rate of F-center primary production. The shape of the growth curve is entirely explained by secondary reactions (interstitial stabilization). 